\title{
Aquaporin-4-Positive Triple-Negative Breast Cancer Presenting with Paraneoplastic Neuromyelitis Optica Spectrum Disorder
}

\author{
Pilar Carrillo ${ }^{a}$ Teresa Gorría ${ }^{b}$ Daniel Santanac Maria Sepulvedac Iban Aldecoad, e, $f$ \\ Blanca Gonzalez-Farréd, 9 Esther Sanfeliu ${ }^{d, g}$ Eduard Mension $^{a} \quad$ Isaac Cebrecos ${ }^{a}$ \\ Olga Martínez-Saez ${ }^{\mathrm{b}}$ Imma Alonso ${ }^{\mathrm{a}, \mathrm{h}} \quad$ Albert Saiz $^{\mathrm{c}, \mathrm{h}}$ \\ anstitute Clinic of Gynaecology, Obstetrics and Neonatology, Hospital Clinic of Barcelona, Barcelona, Spain; ${ }^{b}$ Department \\ of Medical Oncology, Hospital Clinic of Barcelona and Translational Genomics and Targeted Therapeutics in Solid Tumors, \\ August Pi I Sunyer Biomedical Research Institute (IDIBAPS), Barcelona, Spain; 'Neurology Service, Hospital Clinic of Barcelona, \\ and University of Barcelona, Barcelona, Spain; ${ }^{\mathrm{d} D e p a r t m e n t}$ of Pathology, Biomedical Diagnostic Center, Hospital Clinic \\ of Barcelona, Barcelona, Spain; ' Molecular Pathology of Inflammatory Conditions and Solid Tumours, August Pi I Sunyer \\ Biomedical Research Institute (IDIBAPS), Barcelona, Spain; ${ }^{f}$ Neurological Tissue Bank of the Biobanc-Hospital Clinic-IDIBAPS, \\ Barcelona, Spain; ${ }^{9}$ Translational Genomics and Targeted Therapeutics in Solid Tumors, August Pi I Sunyer Biomedical \\ Research Institute (IDIBAPS), Barcelona, Spain; ${ }^{\text {h} A u g u s t ~ P i ~ I ~ S u n y e r ~ B i o m e d i c a l ~ R e s e a r c h ~ I n s t i t u t e ~(I D I B A P S), ~ B a r c e l o n a, ~ S p a i n ~}$
}

\section{Established Facts}

- Neuromyelitis optica spectrum disorder (NMOSD) is an inflammatory disorder of the central nervous system. Although the disorder is most commonly an idiopathic autoimmune condition, it may also occur as a paraneoplastic syndrome in rare instances.

- The pathogenesis of NMOSD is related to the presence of autoantibodies against aquaporin-4 (AQP4), a water channel mainly expressed in the end feet of astrocytes. In the past years, AQP4 expression has also been found in some tumor tissues.

\section{Novel Insights}

- To our knowledge, only one case of paraneoplastic neuromyelitis optica spectrum disorder related to breast cancer and expression of aquaporin- 4 in the tumor has been published.

- We would like to emphasize the importance of keeping a high clinical suspicion of paraneoplastic presentation and of managing these patients in an experienced multidisciplinary team.

\section{Keywords}

Neuromyelitis optica · Aquaporin-4 - Paraneoplastic syndrome Breast cancer $\cdot$ Triple-negative breast cancer

Pilar Carrillo and Teresa Gorría share first authorship.

\section{Abstract}

Introduction: Neuromyelitis optica spectrum disorder (NMOSD) is an inflammatory central nervous system disorder that preferentially affects the optic nerve and the spinal cord. Although NMOSD is more commonly an idiopathic au- karger@karger.com www.karger.com/bmh

Karger $\stackrel{\text { ' }}{5}$ BOPEN ACCESS
(C) 2022 The Author(s)

Published by S. Karger AG, Basel

This is an Open Access article licensed under the Creative Commons Attribution-NonCommercial-4.0 International License (CC BY-NC) (http://www.karger.com/Services/OpenAccessLicense), applicable to the online version of the article only. Usage and distribution for commercial purposes requires written permission.
Correspondence to:

Eduard Mension, mension@ clinic.cat 
toimmune condition associated with antibodies against aquaporin-4 (AQP4)-IgG, the disease may also occur as a paraneoplastic syndrome in rare instances. In these cases, the expression of AQP4 by the tumor is likely the trigger of the autoimmune response. Case Presentation: We describe the case of a 32-year-old woman who presented with progressive tetraparesis, cranial involvement, respiratory failure, and spinal cord MRI compatible with longitudinally extensive transverse myelitis, few days after being diagnosed with a T3N1M0 triple-negative right breast cancer. Due to the history of concurrent breast cancer and after ruling out metastatic spinal cord involvement, the possibility of a paraneoplastic origin was raised. AQP4-IgG were found in the serum and CSF by cell-based assay, confirming the diagnosis of NMOSD. The patient was treated with corticosteroids, plasma exchange, and rituximab. Concomitantly, breast cancer therapy was started with an adapted neoadjuvant chemotherapy scheme based on carboplatin and paclitaxel. An initial slight improvement slowed down; so, a right mastectomy with lymphadenectomy was performed. Expression of AQP4 was demonstrated in the tumor. The patient presented a significant neurological improvement after combined treatment regaining muscular balance and strength in upper and lower extremities. Conclusion: NMOSD may have a paraneoplastic origin associated with breast cancer and the importance of its early detection since the combination of tumoral and immunosuppressive therapy may improve the patient's prognosis.

(C) 2022 The Author(s)

Published by S. Karger AG, Basel

\section{Introduction}

Neuromyelitis optica spectrum disorder (NMOSD) is an inflammatory and demyelinating disorder of the central nervous system (CNS) that preferentially affects the optic nerve and the spinal cord [1]. Most patients have antibodies that target the water channel aquaporin-4 (AQP4)-IgG which is mainly found in the end feet of astrocytes of the CNS [2]. Although NMOSD is more commonly an idiopathic autoimmune condition, the disease may also occur as a paraneoplastic syndrome in rare instances as evidenced by the expression of AQP4 in the underlying tumor [3]. To our knowledge, this is the first case of triple-negative breast cancer associated with paraneoplastic NMOSD and the second one with demonstration of tumor expression of AQP4 published [4]. In this case report, that association will be presented and the importance of early detection will be discussed.

\section{Case Presentation}

Presenting Concerns and Clinical Findings

A 32-year-old Caucasian woman was admitted to our hospital with tetraparesis, cranial nerve involvement, and respiratory failure few days after undergoing a biopsy of a breast nodule that she had detected 2 months earlier in her right breast. A triple-negative breast cancer was diagnosed by the anatomopathological study. Due to the clinical syndrome and the presence of a concurrent breast cancer, the question of a possible paraneoplastic origin was raised.

\section{Diagnostic Focus and Assessment}

The physical examination demonstrated the right breast occupied by a free moving mass with no muscle, nor skin involvement. A mobile lymph node was palpable in the right armpit. The mammography showed an increased density with skin thickening without focal lesions (Fig. 1). The ultrasound showed diffuse skin edema in the right breast, affecting all the quadrants. Two irregular hypoechoic masses were identified in the superior external quadrant and in the union of inferior quadrants without being able to delimitate their margins, precisely. In the axillar region, a group of suspicious nodes were observed with the largest measuring $25 \times 16 \mathrm{~mm}$. In suspicion of a multicentric breast cancer (BIRADS 5), a needle core biopsy of the nodules and an aspiration puncture with fine needle (FNA) of the greatest lymph node were performed. The result was ductal infiltrating carcinoma grade 3 with no vascular invasion nor in situ carcinoma. As for the immunohistochemistry examination, estrogen receptors, progesterone receptors, and HER2 were negative $(0 \%)$, and Ki 67 was $70 \%$. The FNA of the lymph node was positive for metastasis of carcinoma. An extension study consistent of thoracoabdominal scan was carried out without any signs of metastasis.

Three days after being diagnosed with a right triple-negative multicentric T3N1M0 breast cancer, the patient was evaluated in other center because of nausea, vomits, and diarrhea; infection and metastatic causes were ruled out by cultures and chest-abdominal $\mathrm{CT}$, and she was referred to our hospital because of development of paraparesis and trigeminal hypoesthesia. On neurological examination, the patient was alert and oriented, and the cranial nerve examination was normal but for the left trigeminal hypoesthesia. There was a weakness in both legs (MRC right psoas $2 / 5$ and left $3 / 5$ ), preserved tendon reflexes in legs, and bilateral flexor cutaneous plantar reflex. There was a T4-T5 sensory level to light touch and pain, and vibratory and joint position were decreased in the left foot. An urgent spinal cord MRI showed a left lesion involving the dorsal medulla with extension to $\mathrm{C} 1$, a multifocal longitudinally extensive lesion from C7 to D5 and from D9 to D11 without contrast enhancement (Fig. 2). The MRI also showed involvement of the floor of the IV ventricle, which relates to the area postrema syndrome the patient experienced consisting of intractable nausea and vomits which led the patient to seek for medical consult. A lumbar puncture disclosed hyperproteinorrachia $(734 \mathrm{mg} / \mathrm{L})$ and pleocytosis ( $60 \mathrm{cells} / \mathrm{mm}^{3}$; 91\% lymphocytic elements). PCR analysis for an extensive panel of virus was negative, as well as the cultures for infections. Oligoclonal bands and onconeural antibodies were negative but AQP4-IG in serum and CSF were positive [1]. Considering the results of presence of AQP4-IgG in a patient with a longitudinally extensive transverse myelitis (LETM), the diagno-
Carrillo et al. 


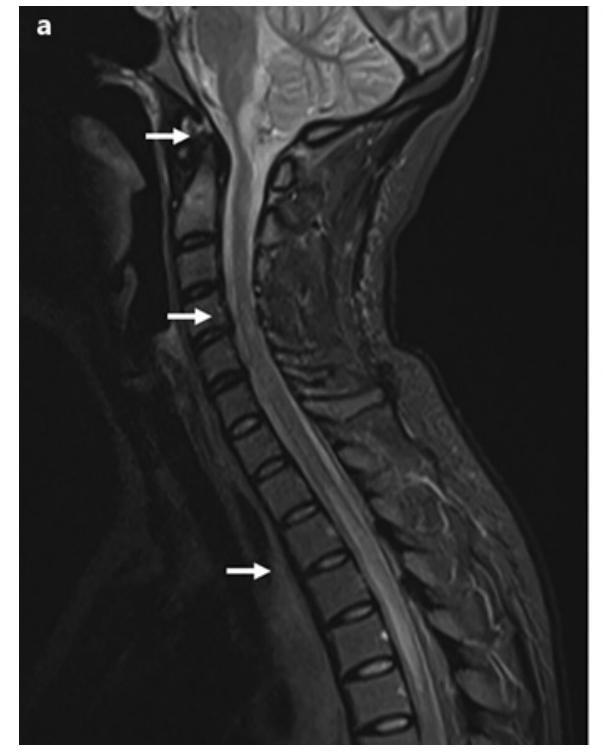

Fig. 1. Brainstem and spinal cord MRI. a Sagittal DP-weighted MRI of the cervical spinal cord shows a dorsal medulla lesion with extension to C1, and C3-C4 and C7-D5 lesions (arrows). b Axial T2-weighted MRI shows in detail the involvement of the left dorsal

sis of NMOSD was established [5], and due to its occurrence in a patient with concomitant breast cancer, the paraneoplastic origin was suspected.

\section{Therapeutic Focus and Assessment}

The patient was admitted in the neurology department and IV methylprednisolone $(1 \mathrm{~g} /$ day $\times 5)$ was started. But a few hours after admission, she had a neurological deterioration with clinical signs of brainstem dysfunction, arm weakness, and paraplegia, followed $12 \mathrm{~h}$ later by episode of acute desaturation (oxygen saturation $82 \%$ ), fever $37.7^{\circ} \mathrm{C}$, and tachypnea requiring intubation and mechanical ventilation. An extubation was attempted, resulting in a reintubation and in the performance of a tracheostomy. Subsequently, the patient maintained spontaneous ventilation but showed signs of drowsiness, anarthria, diplopia, and dysphagia in addition to severe tetraparesis. Both blood cultures and bronchial aspirate tested negative, and IV methylprednisolone therapy was continued for 4 more days, along with a chemotherapy scheme based on carboplatin AUC 1.5 and nab-paclitaxel $125 \mathrm{mg} / \mathrm{m}^{2}$ weekly. After the first chemotherapy cycle, a plasma exchange was started with immunoglobulin reposition every 2 exchanges and a dose of rituximab $(200 \mathrm{mg})$ the day before starting the plasma exchange and a second dose of rituximab ( $1 \mathrm{~g})$ after completion the sixth plasma exchange. Given the concern of a further deterioration without a significant improvement, a radical modified right mastectomy with lymphadenectomy for the removal of the tumor mass was performed 2 weeks after the last chemotherapy cycle. The intention was to eliminate the possible antigenic burden generated by the tumor. The positive expression of AQP4 in the tumor (Fig. 2) confirmed the paraneoplastic origin of the NMOSD.

Triple-Negative Breast Cancer and AQP4Neuromyelitis Optica
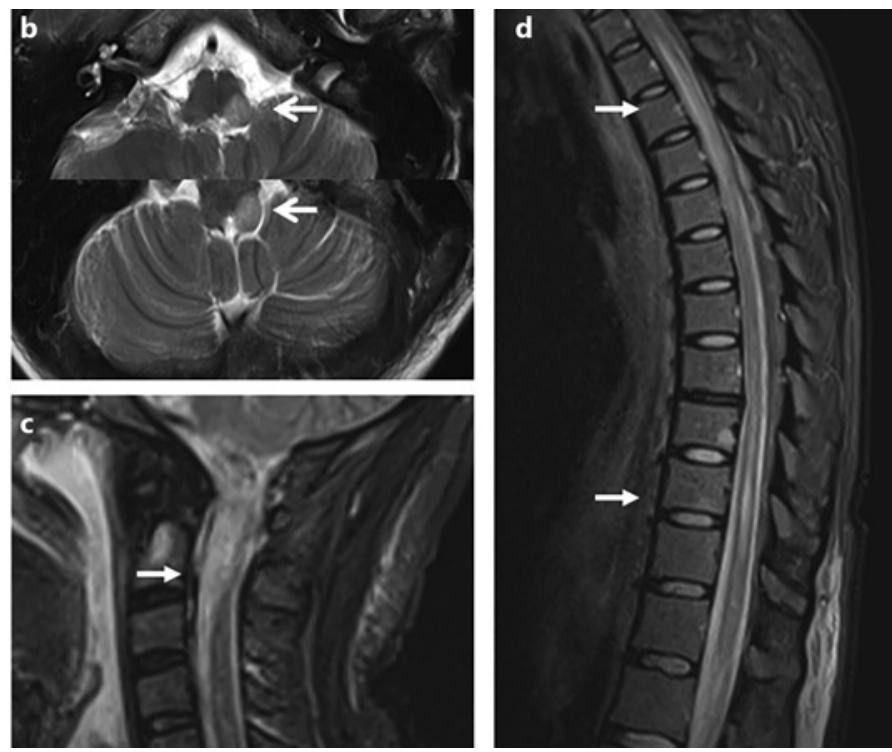

medulla (arrows). c Sagittal T2-weighted MRI shows the extension of the lesion to C2-C3 (arrow). d Sagittal DP-weighted MRI of the thoracic spinal cord shows a longitudinal extensive lesion from $\mathrm{C} 7$ to D5 and from D9 to D11 (arrows).

\section{Follow-Up and Outcomes}

The anatomopathological study showed an invasive multicentric ductal carcinoma, grade 2 , with the largest foci of $32 \mathrm{~mm}$ on the $86 \times 78 \mathrm{~mm}$ tumor bed. There was no evidence of in situ carcinoma or perineural invasion, although images of angiolymphatic invasion were present. The surgical margins were free of disease showing no affectation of the skin, nipple or muscle. The proliferation index Ki 67 had decreased from $70 \%$ to $20 \%$. Nine out of 15 lymph nodes in levels I and II of Berg showed metastasis. No evidence of metastasis was found among the seven level III lymph nodes analyzed. This concluded in an oncological stage ypTNM (AJCC 8th Edition): ypT2(m)ypN2a. Given the patient's young age and the breast cancer subtype being triple negative, a genetic panel study was performed showing no genetic mutations.

During the follow-up, adjuvant weekly chemotherapy was resumed 2 weeks after the surgery with the same scheme previously used until completing 12 cycles of carboplatin-nab-paclitaxel, followed by 4 cycles of epirubicin $90 \mathrm{mg} / \mathrm{m}^{2}$ and cyclophosphamide $600 \mathrm{mg} / \mathrm{m}^{2}$ every 2 weeks. Radiotherapy on the mastectomy bed as well as in the axillary region was also performed. Subsequently, adjuvant capecitabine was provided for a total of 8 cycles.

The patient experienced a progressive neurological improvement. Thus, 1 year later, in treatment with rituximab $(1 \mathrm{~g})$ every 6 months, the muscular balance was almost normal in the upper extremities, she had a slight proximal paraparesis and higher distal weakness (3/5) with Babinski and spasticity; she needed intermittent self-catheterization, and two supports for walking, and remained free of NMOSD relapses. Regarding the patient's cancer status, a second-line chemotherapy treatment consisting of carboplatin and gemcitabine was required, presenting partial metabolic response up to date. 

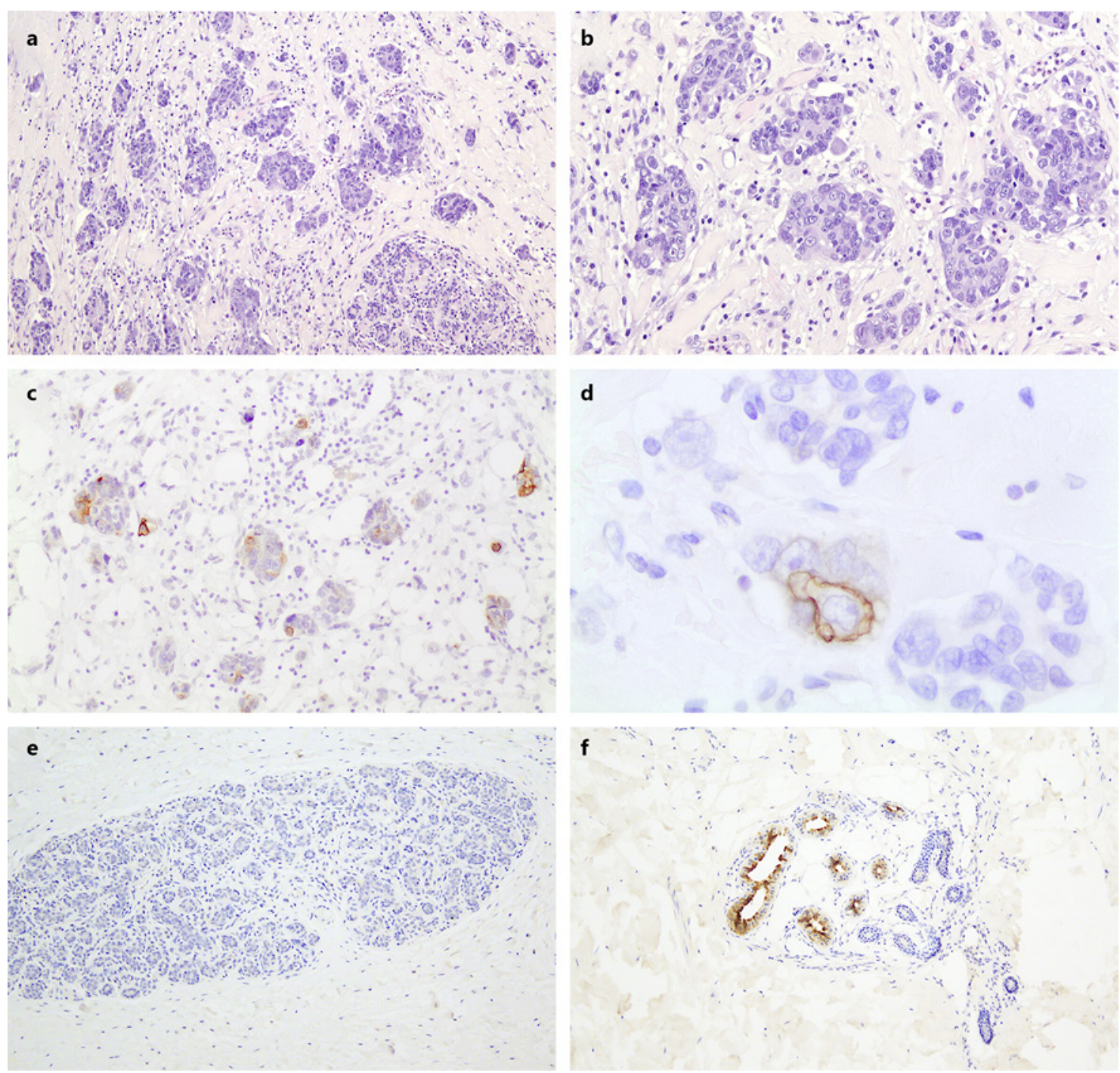

Fig. 2. Pathological findings. a The surgical specimen showed an invasive multicentric ductal carcinoma histological grade 2 alongside remnants of breast parenchyma (bottom right) (hematoxylin and eosin, $\times 100)$. b Infiltrating ductal carcinoma of cells with moderate nuclear pleomorphism in small solid groups (hematoxylin an eosin, $\times 200)$. c AQP4 immunohistochemical staining showed vari-

\section{Discussion}

NMOSD usually presents with relapses of optic neuritis and myelitis, and preferentially, affects young women in their fourth decade of life $[1,6]$. Personal predisposition to suffering other autoimmune diseases such as systemic lupus erythematosus, Sjögren syndrome, or myasthenia gravis is present in almost $25 \%$ of the patients [1].

The cause of the disease is not fully understood, but both genetic and environmental factors may play a role. The dis- able expression with multiple positive cells $(\mathrm{AQP} 4, \times 100)$. d The AQP4 expression was mainly restricted to cell membranes (AQP4, $\times 400)$. e Breast parenchyma was AQP4 negative, while subcutaneous apocrine glands showed moderate expression $(\mathbf{f}, \mathrm{AQP} 4, \times 100)$. Aquaporin-4 ref. A5971 (Sigma) incubated for $20^{\prime}$ at $1 / 400$ on a Leica BOND Max, with $20^{\prime}$ citrate pretreatment.

order is considered as an idiopathic autoimmune inflammatory disease, and almost $70-80 \%$ of the patients have antibodies to AQP4 with direct implication in the pathogenesis of the disease [1,6-8]. AQP4 is the most common water channel in the CNS expressed in the membrane of astrocytes particularly in the foot processes facing microvessels [2]. Thus, the perivascular astrocytes are the target of the immune response, and pathologic studies show a decrease in number of astrocytes, with complement deposits and loss of AQP4 immunoreactivity in the remaining [9]. 
However, in some rare cases NMOSD can have a paraneoplastic origin. Demonstration in some of these patients that the underlying tumor expresses AQP4 suggests that the tumor is the trigger of the autoimmune response. Although different types of cancer have been described in paraneoplastic NMOSD cases associated with AQP4-IgG $[3,4,10-12]$, such as lymphoma $[11,13,14]$, stomach carcinoid [15], esophageal carcinoma $[15,16]$, and teratoma $[17,18]$, the most common are non-small cell lung cancer [3, 19-23] and breast cancer $[3,4,23,24]$, which account for $42 \%$ of all tumors. However, as far as we know, the expression of AQP4 has only been analyzed in one of the 6 cases of breast cancer [4].

In 2015, new criteria were proposed for the diagnosis of neuromyelitis optica and the term of NMOSD was introduced to refer to this entity. In patients with AQP4IgG, the diagnosis of NMOSD include the presence of at least 1 core clinical syndrome related to optic nerve, spinal cord, area postrema, other brainstem, diencephalic or cerebral presentation, and reasonable exclusion of alternative causes [5]. These features were met by our patient.

Up to $40 \%$ of the patients present with an LETM in which the lesion extends over three or more vertebral segments $[1,6-8]$. Therefore, the presentation of LETM in a young female patient in whom AQP4-IgG is detected may lead to believe that the cause is idiopathic, and the search for an undelaying tumor is not considered necessary. This fact is important because in contrast with the current case in whom the diagnosis of the cancer preceded the neurological symptoms, in most paraneoplastic cases $(61 \%)$, the cancer is diagnosed after the onset of the neurological syndrome (median 1 month, range 1-24 months), and a diagnostic delay may have deleterious consequences. The literature review suggests features that may indicate that the risk for an underlying tumor is higher such as the presentation with an area postrema syndrome (intractable nausea and vomiting), and patients older than 45 years, usually male, who present with LETM [3]. Therefore, in those cases, performing a cancer screening would be advisable.

Acute episodes are usually treated with intravenous corticosteroids ( $1 \mathrm{~g} /$ day $\times 3-5$ days). If no response is observed in a few days, additional treatment with plasma exchange is recommended, and some benefits have also been reported after intravenous immunoglobulins [25]. Preventive therapies are usually mycophenolate mofetil and rituximab that appear to be superior to azathioprine [26]. Nevertheless, there is constant research regarding this topic with promising results for other therapies such as eculizumab, satralizumab, and inebilizumab [27].

Although there is no enough scientific evidence regarding the optimal management of these cases and the role of surgery is controversial, some improvement has been described after tumor removal by reducing the antigenic burden [24]. Hence, it was decided to perform surgery before ending the neoadjuvant treatment. A quick improvement of the symptoms was observed, which, without being able to establish a clear causal relationship, suggests that there could be a relationship with the disappearance of the antigenic stimulus as it has been seen in our patient.

\section{Conclusion}

We would like to emphasize the importance of taking into account a possible paraneoplastic origin of NMOSD, especially in those patients who present with AQP4-IgG, and of managing these patients in an experienced multidisciplinary team. In our case, the combination of chemotherapy, immunosuppressing drugs, and surgical removal of the tumor were crucial for the neurological improvement of the patient.

\section{Acknowledgments}

We would like to express our gratitude to all who helped writing the manuscript. Special thanks should go to Professor Saiz, from whose guidance we benefited greatly.

\section{Statement of Ethics}

This paper adheres to the law of data protection and was conducted ethically in accordance with the World Medical Association Declaration of Helsinki. The patient has given written informed consent to publish her case including publication of images.

Ethical approval was not required for this study in accordance with local guidelines (Ethics Committee Hospital Clinic).

\section{Conflict of Interest Statement}

The authors have no conflicts of interest to declare.

\section{Funding Sources}

No funding sources have been used. 


\section{Author Contributions}

Pilar Carrillo and Teresa Gorría were responsible for writing of the case report, the information gathering, and conducting the literature review. They share the first authorship. Daniel Santana was responsible for information gathering. Iban Aldecoa was responsible of AQP4 staining of the tumor and provided the images. Blanca González-Farré and Esther Sanfeliu contributed to gathering information about the tumor and provided images. Eduard Mension and Isaac Cebrecos were responsible for information gathering. Eduard Mension is the corresponding author. Olga Martínez-
Saez and Imma Alonso contributed to information gathering, literature review, and drafting the manuscript. Maria Sepúlveda and Albert Saiz contributed to information gathering, literature review, and drafting the manuscript. Albert Saiz is the senior author.

\section{Data Availability Statement}

All data regarding the case presented are included in this. Further inquiries can be directed to the corresponding author.

\section{References}

1 Sepúlveda M, Armangué T, Sola-Valls N, Arrambide G, Meca-Lallana JE, Oreja-Guevara C, et al. Neuromyelitis óptica spectrum disorders. Comparison according to the phenotype and serostatus. Neurol Neuroimmunol Neuroinflamm. 2016;3:e225.

2 Lennon VA, Wingerchuk DM, Kryzer TJ, Pittok J, Lucchinetti C, Fujihara K, et al. A serum autoantibody marker of neuromyelitis optica: distinction from multiple sclerosis. Lancet. 2004;364:2106-12.

3 Sepúlveda M, Sola-Valls N, Escudero D, Rojc $\mathrm{B}$, Barón M, Hernández-Echevarría L, et al. Clinical profile of patients with paraneoplastic neuromyelitis optica spectrum disorder and aquaporin-4 antibodies. Mult Scler. 2018; 24(13):1753-59.

4 Armağan $H$, Tüzün E, Içöz S, Birisik O, Ulusoy C, Demir G, et al. Long extensive transverse myelitis associated with aquaporin-4 antibody and breast cancer: favorable response to cancer treatment. J Spinal Cord Med. 2012;35:267-9.

5 Wingerchuk DM, Banwell B, Bennett JL, Cabre P, Carroll W, Chitnis T, et al. International consensus diagnostic criteria for neuromyelitis optica spectrum disorders. Neurology. 2015;85:1-13.

6 Sepulveda M, Aldea M, Escudero D, Llufriu S, Arrambide G, Otero-Romero S, et al. Epidemiology of NMOSD in Catalonia: influence of the new 2015 criteria in incidence and prevalence estimates. Mult Scler. 2018;24(14): 1843-51.

7 Beauchemin P, Iorio R, Traboulsee AL, Field T, Tinker AV, Carruthers RL. Paraneoplastic neuromyelitis optica spectrum disorder: a single center cohort description with two cases of histological validation. Mult Scler Relat Disord. 2018 Feb;20:37-42.

8 Shahmohammadi S, Doosti R, Shahmohammadi A, Azimi A, Sahraian MA, Fattahi MR, et al. Neuromyelitis optica spectrum disorder (NMOSD) associated with cancer: a systematic review. Mult Scler Relat Disord. 2021 Aug 27;56:103227.
9 Lucchinetti CF, Mandler RN, McGavern D, Bruck W, Gleich G, Ransohoff R, et al. A role for humoral mechanisms in the pathogenesis of Devic's neuromyelitis optica. Brain. 2002; 125:1450-61.

10 Pittock SJ, Lennon VA. Aquaporin-4 autoantibodies in a paraneoplastic context. Arch Neurol. 2008;65:629-32.

11 Blackburn K, Wang C, Greenberg B. Two cases of aquaporin-4 positive neuromyelitis optica associated with T-cell lymphoma. J Neuroimmunol. 2020;338:577092.

12 De Santis G, Caniatti L, de Vito A, de Gennaro R, Granieri E, Tola MR. A possible paraneoplastic neuromyelitis optica associated with lung cancer. Neurol Sci. 2009;30:397400.

13 Wang Y, Miocinovic S, Greenberg BM. Neuromyelitis optica spectrum disorder associated with autoimmune hemolytic anemia and lymphoma. Neurologist. 2015;20:33-4.

14 Nakayama-Ichiyama S, Yokote T, Hiraoka N, Iwaki N, Takayama A, Kobayashi K, et al. A paraneoplastic neuromyelitis optica spectrum disorder associated with a mature B-cell neoplasm. Leuk Res. 2011;35:e111-3.

15 Kon T, Ueno T, Suzuki C, Nunomura J, Igarashi S, Sato T, et al. Aquaporin-4 antibody positive neuromyelitis optica spectrum disorder associated with esophageal cancer. J Neuroimmunol. 2017;309:38-40.

16 Wiener DC, Kaplan TB, Bravo-Iñiguez CE, Miller J, Berkowitz AL, Jaklitsch MT. Paraneoplastic neuromyelitis optica spectrum disorder as presentation of esophageal adenocarcinoma. Ann Thorac Surg. 2018;105:e133-5.

17 Frasquet M, Bataller L, Torres-Vega E, Durán-Moreno M, García-Moreno JM, Sevilla $\mathrm{T}$, et al. Longitudinally extensive transverse myelitis with AQP4 antibodies revealing ovarian teratoma. J Neuroimmunol. 2013; 263:145-7.
18 Bernard-Valnet R, Cobo-Calvo A, Siegfried A, Marasescu R, Bonnan M, Ballan G, et al. Paraneoplastic neuromyelitis optica and ovarian teratoma: a case series. Mult Scler Relat Disord. 2019;31:97-100.

19 Verschuur CV, Kooi AJ, Troost D. Anti-aquaporin 4 related paraneoplastic neuromyelitis optica in the presence of adenocarcinoma of the lung. Clin Neuropathol. 2015;34:232-6.

20 Iorio R, Rindi G, Erra C, Damato V, Ferilli M, Sabatelli M. Neuromyelitis optica spectrum disorder as a paraneoplastic manifestation of lung adenocarcinoma expressing aquaporin-4. Mult Scler. 2015 May;21(6):791-4.

21 Deuel LM, Bunch ME. A case of paraneoplastic neuromyelitis optica associated with small cell lung carcinoma. J Neuroimmunol. 2018; 316:130-2.

22 Baik KW, Kim SH, Shin HY. Paraneoplastic neuromyelitis optica associated with lung adenocarcinoma in a young woman. J Clin Neurol. 2018;14:246-7.

23 Fang W, Zheng Y, Cai MT, Zhang YX. Neuromyelitis optica spectrum disorder with lung adenocarcinoma and intraductal papillary mucinous neoplasm. Mult Scler Relat Disord. 2019;32:77-80

24 Weijin L, Cheng L, Yulan T, Fan H, Huimin $\mathrm{K}$, Shuolin L, et al. Aquaporin-4 antibody positive short transverse myelitis associated with breast cancer. Mult Scler Relat Disord. 2019; 30:119-22.

25 Moore RR, Tanner M. The latest diagnostic criteria and treatment options for neuromyelitis optica. JAAPA. 2019;32(7):1.

26 Huang W, Wang L, Zhang B, Zhou L, Zhang T, Quan C. Effectiveness and tolerability of immunosuppressants and monoclonal antibodies in preventive treatment of neuromyelitis optica spectrum disorders: a systematic review and network meta-analysis. Mult Scler Relat Disord. 2019;35:246.

27 Levy M, Fujihara K, Palace J. New therapies for neuromyelitis optica spectrum disorder. Lancet Neurol. 2021;20(1):60-7. 